\title{
How wet can you get?
}

Some concepts are useful but difficult to quantify, as Philip Ball illustrates with the property of hydrophobicity.

Y ou're designing a microfluidic network, and if you can controllably switch how fully water wets the channels, you can direct the flows at will.

Or: you're designing a ligand to bind to a protein, and you want to tailor its surface to compete with hydration in the protein's active site.

Or: you've got to use a particular surface coating for a biomedical device, but you suspect that with the right choice of micropatterning you can tune the adhesive properties to reduce biofouling by bacteria.

In all of these cases, you're juggling with the property of hydrophobicity: the degree to which water will wet the surface in question. It would be great to have a way of calculating how the function you're after depends on the hydrophobicity of the surface.

You'd like a parameter that quantifies this property.

And here's the problem: they already exist - in the plural. There is not a single measure of hydrophobicity, but several. Hydrophobicity is one of those quantities that seem so often to arise in the chemical sciences, which one can grasp intuitively but are frustratingly hard to turn into rigorous, quantitative concepts. As Granick and Bae put it, "Like great art, everyone recognizes hydrophobicity but few agree on the details"

At first glance, nothing could seem easier. You can define the hydrophobicity of a material in terms of the internal contact angle that a water droplet makes with its surface. A perfectly hydrophobic surface is totally waterrepellent, with a contact angle of $180^{\circ}$, while a perfectly wettable (hydrophilic) surface has a contact angle of zero.

Contact angles are in principle easy to measure, and have a straightforward interpretation in terms of the balance of surface free energies for the three interfaces of water, air and solid, as expressed in Young's equation. But the situation gets more complicated once you consider dynamics often the practically relevant case. It has long been known that surface defects can 'pin' the advancing and receding edges of a droplet to make the contact angles respectively greater and smaller than their equilibrium values. Which value then is the most salient?

Pinning can be caused by microscopic topographical irregularities, which is to say, surface roughness. Water might penetrate right down into the valleys - known as Wenzel wetting - or it might bridge asperities while bubbles of air remain beneath, known as Cassie-Baxter wetting. The bulk contact angles are different in the two cases, being described by modified forms of the Young equation. Cassie-Baxter wetting is found in nature, for example on lotus leaves and the body of the water strider. It offers a way to make 'superhydrophobic' surfaces in which the intrinsic hydrophobicity of the material is enhanced by micropatterning ${ }^{2}$. The peculiar nature of water itself can spring surprises. Metals are generally considered hydrophilic because of bonding between water's oxygen lone pairs and the surface metal atoms. But on a $\mathrm{Pt}(111)$ surface, a water monolayer is ordered in a way that leaves no free, uncoordinated $\mathrm{OH}$ groups at the surface available for hydrogen bonding. Odd as it may sound, this water monolayer is thus itself hydrophobic ${ }^{3,4}$.

Many real surfaces are not just bumpy but chemically inhomogeneous: patchy, more hydrophobic in some parts than others. The wetting properties then can be subtle and non-intuitive. Chemical inhomogeneity is particularly germane to the important case of protein surfaces, which are often represented as disorderly patchworks of hydrophobic and hydrophilic regions. In the crude picture of protein folding, insoluble, hydrophobic residues such as leucine aggregate in the interior, while polar or hydrogen-bonding hydrophilic residues such as arginine stay on the surface.

The truth is more complicated: pockets of water can be trapped inside, while the surfaces can have substantial regions of hydrophobicity. Both of these are often functionally important. Hydrophobic surfaces stick together in water - a phenomenon called hydrophobic attraction, which is surprisingly hard to understand fully - and this interaction can direct the assembly of proteins into functional multi-protein units. More generally, the hydrophobicity of the surface affects the mobility and structure of water in the hydration shell, which can feed back into aspects of protein function.

All this has prompted a desire to develop hydrophobicity scales that quantify this property for different residues - as seen in the pictured example, where individual amino acids have been assigned a value of hydrophobicity that depends on their chemical composition, represented on a 'rainbow' scale running from red (strongly hydrophilic) to blue (strongly hydrophobic). But it now seems that a wholly reductionist approach is too simplistic: a residue's hydrophobicity may depend on its context, that is, on the nature of its neighbours in the folded state ${ }^{5}$. Hydrophobicity thus becomes an emergent property, albeit still somewhat localized.

How does one navigate these complexities for designing wetting and hydration properties of molecules and materials? Not, evidently, by hoping that they can be captured in a single number. For proteins, and presumably for other, synthetic macromolecules, it has been proposed that hydrophobicity be seen as a multi-dimensional property. For more extended surfaces, hydration may be qualitatively different at different length scales ${ }^{6}$, and distinctly non-additive for chemically non-uniform surfaces. In short, we need many definitions of what it means to be wet.

PHILIP BALL is a science journalist based in London. e-mail:p.ball@btinternet.com

\section{References}

1. Granick, S. \& Bae, S. C. Science 322, 1477 (2008).

2. Marmur, A. Soft Matter 9, 7900-7904 (2013).

3. Wang, C. et al. Phys. Rev. Lett. 103, 137801 (2009)

4. Limmer, D. T., Willard, A. P., Madden, P. \& Chandler, D. Proc. Natl Acad. Sci. USA 110, 4200-4205 (2013).

5. Patel, A. J. \& Garde, S. J. Phys. Chem. B 118, 1564-1573 (2014).

6. Chandler, D. Nature 437, 640-647 (2005).

$\mathrm{m} e \AA \mathrm{s} \mathrm{\textrm {s }} \mathrm{E}_{\mathrm{h}}$

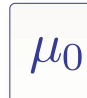

$\alpha$

